\title{
The Development and Evaluation of a Novel Thoracoscopic Diaphragmatic Hernia Repair Simulator
}

\author{
Katherine A. Barsness, MD, Deborah M. Rooney, $\mathrm{PhD}$, ${ }^{2}$ and Lauren M. Davis, BA ${ }^{3}$
}

\begin{abstract}
Background: Thoracoscopic diaphragmatic hernia $(\mathrm{DH})$ repair has a high recurrence rate. Effective simulation modeling may improve technical performance for thoracoscopic DH repair. The study purpose was to evaluate measures of validity evidence for a low-cost thoracoscopic DH simulator.

Materials and Methods: Synthetic diaphragm/intestine was connected to a to-scale left-sided neonatal rib cage and then covered with synthetic skin. Forty participants evaluated the DH repair simulator, using survey ratings that were analyzed for test content and internal structure validity evidence.

Results: Observed averages (on a scale of 0-5) for the domains were 4.6 for Relevance, 4.5 for Value, 4.3 for Physical attributes, 4.0 for Realism of experience, 3.8 for Realism of materials, and 4.2 for Ability to perform. The highest observed averages were "relevance to practice" and "value, testing tool," whereas the lowest ratings were realism, intestines and realism, diaphragm. The observed average of global opinion ratings was 2.84/4.0, indicating the simulator can be considered for teaching thoracoscopic DH repair but could be improved. Experienced surgeons (more than eight DH repairs, at least four thoracoscopic repairs) had higher overall ratings than inexperienced surgeons (4.4 versus $4.1, P=.001$ ).

Conclusions: We successfully created a thoracic space relevant to a thoracoscopic DH repair and completed the model using inexpensive, readily accessible materials. After participants performed a simulated thoracoscopic DH repair, their ratings indicated the simulator was relevant to clinical practice and valuable as a learning tool but still requires improvements. Improvements and additional evaluation of validity evidence (content, internal structure, response processes, and relationship to other variables) are necessary prior to full implementation of this model as an educational tool.
\end{abstract}

\section{Introduction}

$\mathbf{W}$ ITH WIDESPREAD ADOPTION OF ADVANCED minimally invasive techniques, several pediatric surgeons have begun performing minimally invasive repair of congenital diaphragmatic hernias (DHs). Unfortunately, several authors have noted a higher than predicted risk of a recurrent hernia after a minimally invasive repair. ${ }^{1-3}$ A recent metaanalysis found that the relative risk of recurrence after a minimally invasive DH repair is 3.2 (95\% confidence interval 1.1-9.3) compared with an open procedure. ${ }^{4}$ The Congenital Diaphragmatic Hernia Study Group found similar results with an adjusted odds ratio of 3.59 (95\% confidence interval 1.92-6.71) for recurrence after a minimally invasive DH repair. ${ }^{5}$
The etiology of the higher recurrence rates with minimally invasive DH repair is believed to be secondary to key technical differences in the operation, most notably in the limited ability to mobilize the inferior rim of the diaphragm during the thoracoscopic approach. ${ }^{4,5}$ Standardization of technique to mirror those centers with lower recurrence rates may decrease the impact of this and other technical considerations. However, several authors have also postulated that an inherent learning curve exists for the thoracoscopic approach and that the early studies may be demonstrating that learning curve. $^{2-5}$

In an era of increased scrutiny of patient-centered outcomes, creative opportunities need to be explored to remove the learning curve from the operating room. No longer is it accepted that a surgeon who is new to a particular technique

\footnotetext{
${ }^{1}$ Division of Pediatric Surgery, Ann and Robert H. Lurie Children's Hospital of Chicago, Northwestern University Feinberg School of Medicine, Chicago, Illinois.

${ }^{2}$ Department of Medical Education, University of Michigan Medical School, Ann Arbor, Michigan

${ }^{3}$ Center for Simulation Technology and Immersive Learning, Northwestern University Feinberg School of Medicine, Chicago, Illinois.
} 
should have a higher rate of complications than a surgeon experienced in that same technique. ${ }^{6}$ Surgical experience is a complex combination of cognitive and technical skills, acquired through exposure to surgical disease. Unfortunately, surgeon exposure to DHs is decreasing across the nation. In 1995, North American trainees performed a mean of $11 \mathrm{DH}$ repairs. That number dropped to less than seven in $20066^{7,8}$ Perhaps more concerning is that the average practicing pediatric surgeon is performing fewer than two DH repairs per year. ${ }^{8}$ These data, similar across several congenital anomalies, form the basis for developing relevant simulation models for education.

Surgical simulation attempts to recreate the operating room environment authentically, providing opportunities for feedback and time for deliberate practice. Advanced or highfidelity simulation may have the added benefit of decreasing operative learning curves for key technical skills., ${ }^{9,10}$ Unfortunately, few opportunities for high-fidelity simulation education exist in pediatric surgery. ${ }^{11}$ To that end, we set out to create a low-cost, realistic simulation model that replicated key features of a thoracoscopic DH repair. The purpose of this pilot study was to evaluate early validity evidence of test content and internal structure for a simulated thoracoscopic DH repair.

\section{Materials and Methods}

\section{Study setting}

After review and exempt approval by the Ann and Robert H. Lurie Children's Hospital of Chicago Institutional Review Board (protocol number 2012-15098), data were collected during a national pediatric surgery training course for advanced minimally invasive surgery. Thirty-four of 40 pediatric surgery residents and 6 of 12 faculty pediatric surgeons performed on the thoracoscopic DH repair simulator and agreed to fill out an evaluation form. Participants were divided into "experienced" and "novice" groups, based on self-reported experience with thoracoscopic DH repair. Nine experienced surgeons reported a mean of 16 (range, 8-30) total DH repairs, with a mean of 7 (range, 4-10) thoracoscopic $\mathrm{DH}$ repairs. Thirty-one novice surgeons reported a mean of 6 (range, 0-15) total DH repairs, with a mean of less than 1 (range, 0-3) for thoracoscopic DH repair.

\section{Simulator}

Accurate measurements of ribs, thoracic space, and scapulae for term neonates (50th percentile for age) were obtained from literature review. Solidworks 3D modeling software (Dassault Systèmes SolidWorks Corp., Waltham, MA) was used to design a rib cage with scapulae, replicating the exact dimensions of the thoracic cavity of a neonate. The rib cage was printed in acrylonitrile-butadiene-styrene plastic on rapid prototyping machinery. Because repair of a $\mathrm{DH}$ is more commonly performed through the left chest, only the left side of the chest was printed. A base of platinum-cured silicon rubber was created to stabilize the rib cage. The artificial diaphragm with a posterior lateral defect was secured to the ribcage with eyehooks around the 11th rib, and artificial intestines were herniated through the defect in the diaphragm (Fig. 1). The entire apparatus was covered with a synthetic silicon rubber skin.

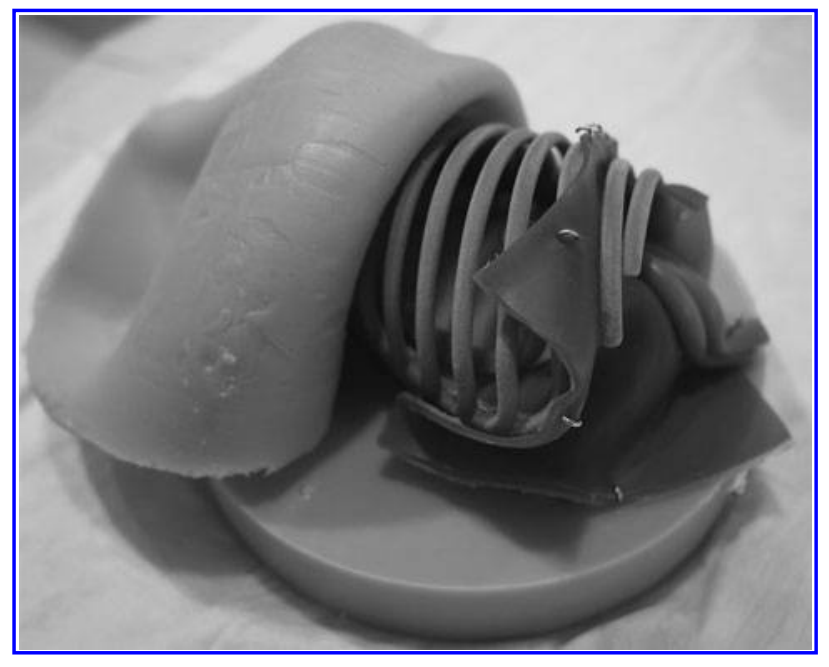

FIG. 1. Thoracoscopic diaphragmatic hernia simulator, with skin pulled back to illustrate ribcage, diaphragm, and intestines.

\section{Survey and rating procedures}

A 20-item survey used to rate the simulator consisted of 19 5-point rating scales and one 4-point global rating scale. The first 19 items were used to rate the simulator across six domains: (a) Physical attributes, (b) Realism of materials, (c) Realism of experience, (d) Ability to perform task, (e) Value, and (f) Relevance. The last item was used to capture participants' overall impression of the simulator.

The first three domains (Physical attributes, Realism of materials, and Realism of experiences) were scored as "Not at all realistic" (1), "Lacks too many features to be useful" (2), "Don't know" (3), "Adequate realism, but could be improved" (4), and "Highly realistic, no changes needed" (5). The fourth domain (ability to perform tasks on the new simulator) was scored as "Too difficult to perform" (1), "Very difficult to perform" (2), "Difficult to perform" (3), "Somewhat easy to perform" (4), and "Very easy to perform" (5). The fifth domain (value) was scored as "No value" (1), "Little value" (2), "Don't know" (3), "Some value" (4), and "Great deal of value" (5). The sixth domain (relevance to practice) was scored as "No relevance" (1), "Little relevance" (2), "Don't know" (3), "Some relevance" (4), and "Great deal of relevance" (5). The global rating item was scored on a 4-point rating scale.

\section{Analyses}

Evidence relevant to test content. To evaluate validity evidence relevant to test content, we used an application from modern test theory: a Rasch model. Analysis was performed using the Facets software version 3.68 (Linacre, 2011; Winstep, Beaverton, OR). For this study, we used a many-facet Rasch model consisting of three facets (participants $\times$ role $\times$ items) to acquire three indices used to evaluate content validity: observed averages, item-measure correlation, and item outfit statistics. These indices, described in greater detail by Wolfe and Smith, ${ }^{12}$ are summarized as follows.

Observed averages. The observed average for each of the 20 items indicates the participants' averaged ratings. Higher 
observed averages suggest high perceived value for that particular characteristic of the simulator, whereas lower observed averages suggest lower perceived value.

Point-measure correlations. The point-measure correlation, also called the item-measure correlation, provides a Pearson correlation between the vector of scores on an item and the perceived value of the simulator. The correlation identifies the degree in which the scores on an item are consistent with the averaged scores of the remaining items. A positive point-measure correlation is ideal and indicates that the particular item contributed useful information to the construct measured by the test as a whole. For this application, a negative value for a particular item may suggest that the item may be measuring a different construct than the other items and fail to offer evidence of content validity.

Item outfit standardized and mean-square statistics. In order to evaluate evidence of content validity, we reviewed item outfit standardized and mean-square statistics. As described by Wright and Masters, ${ }^{13}$ standardized outfit statistics (Z-standardized) are $t$ tests of the hypothesis "Do the data fit the model perfectly, with an expected value of 0.0." Acceptable values typically range from -2.0 to 2.0. Negative values indicate a high level of rating agreement, whereas values over 2.0 indicate a low level of rating agreement. Similar to Z-standardized statistics, outfit mean-square values greater than 2.0 suggest that responses do not fit the particular model and can distort or degrade the measurement system if included in an analysis. In this study, we considered the existence of a high number (over $5 \%$ ) of items with outfit Z-standardized and mean-square values higher than 2.0 a threat to content validity. For our purposes, we have extended this application to test "relevance and representativeness" of simulator characteristics.

Evidence relevant to internal structure. To evaluate evidence of internal structure, we used a traditional method based on classical test theory and evaluated internal consistency as estimated by Cronbach's alpha. On a possible range of 0.0 to 1.0 , a low internal consistency estimate suggests decreased agreement in participants' ratings and fails to support evidence of internal structure.

\section{Results}

\section{Material costs}

The cost to print and assemble one simulator was \$218 US dollars. Broken down by individual parts, the ribcage was $\$ 200$, the intestines and diaphragm were $\$ 2$, and the skin and base were $\$ 8$ each. For 40 participants rotating through six stations, the total cost was \$1408 US dollars to have a simulator at each station and replacement skins for the duration of the course.

\section{Observed averages}

There were no overall differences in observed averages when comparing resident and faculty surgeons (4.3 versus $4.1, P=.22)$. However, when experienced and novice groups were compared, experienced surgeons had higher overall ratings compared with novice surgeons (4.4 versus 4.1,
$P=.001)$. In descending order, the observed averages of the six domains were 4.5 (Relevance), 4.3 (Physical attributes), 4.0 (Realism of experience), 3.8 (Realism of materials), 4.2 (Ability to perform task), and 4.3 (Value). With observed averages greater than 4.4, the three highest-rated items were Physical attributes-Chest circumference, Physical attributes-Chest depth, and Value of simulator as training tool, whereas the lowest ratings were 3.5 (Realism of materials-Herniated intestines), 3.8 (Realism of materials-Diaphragm), and 3.9 (Realism of materials-Skin) (Table 1). These results support validity evidence relevant to test content. The observed average of global opinion ratings was 2.8 out of a maximum of 4 , indicating that, overall, participants believed the current thoracoscopic $\mathrm{DH}$ repair simulator could be considered for use in pediatric surgery education, but improvements were recommended.

\section{Point-measure correlations}

All items have positive point-measure correlations, ranging between 0.29 and 0.78 . Positive point-measure correlations for the 20 items suggest that these items contribute to a single construct and offer evidence of content validity.

\section{Item outfit standardized and mean-square statistics}

Review of item fit statistics indicated that "Value as a testing tool" had an outfit Z-standardized statistic of 3.6. In itself, this might indicate that this particular item may not fit

Table 1. Observed Averages for the 20-Item SURVEY INSTRUMENT

\begin{tabular}{lc}
\hline Domain, item & Rating (out of 5) \\
\hline Physical attributes (average $=4.32)^{\mathrm{a}}$ & \\
1. Chest circumference & 4.42 \\
2. Chest depth & 4.47 \\
3. Intercostal space & 4.39 \\
4. Landmark tactility, scapula & 4.26 \\
5. Landmark visualization, scapula & 4.26 \\
6. Orientation of DH defect & 4.09 \\
Realism of materials (average $=3.84)^{\mathrm{a}}$ & \\
7. Skin & 3.88 \\
8. Rib flexibility & 4.18 \\
9. Diaphragm & 3.83 \\
10. Herniated intestines & 3.50 \\
Realism of experience (average $=4.00)^{\mathrm{a}}$ & \\
11. Location of defect & 4.05 \\
12. Suturability of diaphragm & 4.00 \\
13. Represent expected experience & 3.95 \\
Ability to perform tasks (average $=4.24)^{\mathrm{a}}$ & \\
14. Proper placement of port sites & 4.34 \\
15. Reduction of herniated intestine & 4.29 \\
16. Closure of diaphragmatic defect & 4.08 \\
Value (average =4.42) & \\
17. Value as training tool & 4.54 \\
18. Value as testing tool & 4.30 \\
Relevance & \\
19. Relevance to practice & 4.57 \\
Overall rating & \\
20. Global assessment & \\
\hline
\end{tabular}

${ }^{a}$ Where 4 represents adequate realism as is but could be improved. $\mathrm{DH}$, diaphragmatic hernia. 
the construct. However, on closer examination, its higher mean-square fit statistic of 2.37 indicated this item is likely associated with higher than expected variance and noise. Basically, this suggests that there were some outlying extreme responses that did not agree with the rest of the participants' ratings. On the other extreme, "Ability to perform port placement" had an outfit Z-standardized statistic of -2.3 and a mean square statistic of -2.1 , indicating that this item has so much agreement that there is less variability of rating than expected. However, because our observed averages were relatively high, this does not threaten construct validity evidence. Inter-item consistency was 0.91 estimated by Cronbach's alpha. The high degree of consistency across different items offers support of evidence of internal structure.

\section{Discussion}

We present our data on a low-cost simulation model for thoracoscopic DH repair. The initial validation data suggest that the simulator is relevant to clinical practice and has value as a learning tool. However, improvements in the model could be considered prior to implementation of the current simulator as an educational tool.

Traditionally, when validity evidence is reported, researchers turn to the Standards for Educational and Psychological Testing (Standards). ${ }^{14}$ The current Standards identified five different sources of validity evidence: (a) test content, (b) internal structure, (c) response processes, (d) relationships to other variables, and (e) consequences of testing. For the purpose of this pilot study, we focused on the evaluation of validity evidence relevant to test content and internal structure.

Our strongest physical attributes of the simulator were chest circumference and chest depth. These physical attributes met our expectations, given that the thoracic space was created according to specific measurements of a neonatal chest. The weakest attributes were the realism of the tissues, including skin, diaphragm, and intestine. Although we were able to create these tissues using low-cost synthetic materials, we did not realistically recreate the entire operative experience of a thoracoscopic DH repair. Comments were consistent with poor haptic feedback of the synthetic materials. We have previously published our experience with a real tissue thoracoscopic esophageal atresia/tracheoesophageal fistula repair simulator, completed with second-trimester fetal bovine tissue. ${ }^{15,16}$ In the thoracoscopic esophageal atresia/ tracheoesophageal fistula model, our Realism of materials and Realism of experience were rated higher than in this study using synthetics. Unfortunately, the cost of real tissue will drive the cost for the simulator far higher. Future studies evaluating the relative advantages of real tissue versus simulated tissue in the $\mathrm{DH}$ simulator will have to take into account the added expense of the real tissue.

It is notable that those participants with more experience with thoracoscopic DH repair had higher overall observed averages compared with novices of the technique. These data illustrated that, although haptic feedback of the tissues is an issue, the current simulator does a good job of replicating the motions and technical considerations of a thoracoscopic DH repair. Even more interesting is that the current simulator's highest observed averages were in "Relevance to practice" and "Value as a testing tool." The concept of using simulation as an instrument to test competence is only beginning to be explored by the American Board of Surgery. For the first time ever, certification in General Surgery requires completion with passing scores on Fundamentals of Laparoscopic Surgery, a broadly accepted program for measuring technical skill in general surgeons. ${ }^{17}$ In fact, the current class of pediatric surgery trainees is among the first class in which Fundamentals of Laparoscopic Surgery is a requirement for board certification. Perhaps this recent experience of the majority of the study participants is why "Value as a testing tool" is ranking so highly. The role for pediatric-specific instruments used to measure technical skill in pediatric surgery board certification cannot be determined at this time, as the validation evidence for such instruments is not available. This study and others like it is among the first of many steps toward validated measures from pediatric-specific simulation instruments.

There are several limitations related to the interpretation and applications of the findings from this study. First, these data were collected during a course on advanced minimally invasive surgical techniques for pediatric surgical trainees. Although the participants were from several different institutions, they consisted primarily of trainees. The trainee sample had a relatively narrow range of experience, which may have decreased the variability of some ratings. Expansion of the study to various levels of pediatric surgical experience would not only increase the sample size, but could also improve variability of ratings. Second, the overall global rating was not as high as anticipated. As indicated previously, we likely need to move toward tissue with haptic feedback more consistent with neonatal tissues. Future studies will include a real tissue simulator, compared with the current version. Finally, we have only evaluated validity evidence relative to test content and internal structure. With this pilot study, we have not begun to explore evidence relevant to response processes, relationships to other variables, and consequences of testing.

Despite these limitations, the future potential of validated measures from instruments for simulation in pediatric surgery is significant. The opportunity to practice to proficiency in a simulation lab instead of the operating room has the potential to protect our most fragile subsets of infants from the inherent risks of learning curves for new techniques or procedures. Simulation-based education can also be a key platform in curricula development in the modern era of decreased work hours and decreasing exposure to congenital anomalies. Only with validated measures can we even begin to test these hypotheses of improved outcomes and safer operations for infants and children, as well as more efficient education for our trainees.

In summary, we have created a novel simulation model for thoracoscopic DH repair. The current simulation model is inexpensive to create and use. Despite poor haptic feedback of the tissues, the simulator is highly valued and thought to be relevant to pediatric surgery practice. Initial validity evidence relevant to test content and internal structure supports further structural refinement and subsequent validation of measures from a refined model for use in pediatric surgical simulation.

\section{Acknowledgments}

We would like to acknowledge the Center for Education in Medicine, Northwestern University Feinberg School of Medicine, for development support of the thoracoscopic 
diaphragmatic hernia repair simulator. In addition, we would like to thank Melissa Meyer, BS, for all of her hard work with the data management and processing.

\section{Disclosure Statement}

Provision patent application number is $61 / 818,232$, filed May 1, 2013. There are no financial interests at this time; however, a patent that includes this simulation model has been filed.

\section{References}

1. Gander JW, et al. Early recurrence of congenital diaphragmatic hernia is higher after thoracoscopic than open repair: A single institutional study. J Pediatr Surg 2011;46:1303-1308.

2. Cho SD, et al. Analysis of 29 consecutive thoracoscopic repairs of congenital diaphragmatic hernia in neonates compared to historical controls. J Pediatr Surg 2009;44:80-86; discussion 86.

3. Szavay PO, et al. Perioperative outcome of patients with congenital diaphragmatic hernia undergoing open versus minimally invasive surgery. I Laparoendosc Adv Surg Tech A 2012;22:285-289.

4. Lansdale $\mathrm{N}$, et al. Neonatal endosurgical congenital diaphragmatic hernia repair: A systematic review and metaanalysis. Ann Surg 2010;252:20-26.

5. Tsao K, et al. Minimally invasive repair of congenital diaphragmatic hernia. J Pediatr Surg 2011;46:1158-1164.

6. Healey P, Samanta J. When does the 'learning curve' of innovative interventions become questionable practice? Eur J Vasc Endovasc Surg 2008;36:253-257.

7. Rowe MI, Courcoulas A, Reblock K. An analysis of the operative experience of North American pediatric surgical training programs and residents. J Pediatr Surg 1997;32:184-191.

8. Somme $S$, et al. Alignment of training curriculum and surgical practice: Implications for competency, manpower, and practice modeling. Eur J Pediatr Surg 2012;22:74-79.

9. Palter VN, et al. Ex vivo technical skills training transfers to the operating room and enhances cognitive learning: A randomized controlled trial. Ann Surg 2011;253:886-889.
10. Sturm LP, et al. A systematic review of skills transfer after surgical simulation training. Ann Surg 2008;248: 166-179.

11. Lasko D, Zamakhshary M, Gerstle JT. Perception and use of minimal access surgery simulators in pediatric surgery training programs. J Pediatr Surg 2009;44:1009-1012.

12. Wolfe EW, Smith EV Jr. Instrument development tools and activities for measure validation using Rasch models: Part I-Instrument development tools. I Appl Meas 2007;8: 97-123.

13. Wright BD, Masters GN. Verifying variables and supervising measures. In: Rate Scale Analysis, Rasch Measurement. Chicago, IL: Mesa Press, 1982.

14. American Educational Research Association, American Psychological Association, and National Council on Measurement in Education. Standards for educational and psychological testing. Washington, DC: American Educational Research, 1999.

15. Davis LM, Barsness KA, Rooney DM. Design and development of a novel thoracoscopic tracheoesophageal fistula repair simulator. Stud Health Technol Inform 2013;184: 114-116.

16. Barsness KA. Collaboration in simulation: The design and development of a novel thoracoscopic esophageal atresia/ tracheoesophageal fistula repair simulator. J Pediatr Surg 2013 (in press). DOI:10.1016/j.jpedsurg.2013.03.015.

17. American Board of Surgery. Resident Assessments. 2012. www.absurgery.org/default.jsp?certgsqe_resassess (accessed March 27, 2013).

Address correspondence to: Katherine A. Barsness, MD Division of Pediatric Surgery Ann and Robert H. Lurie Children's Hospital of Chicago 225 East Chicago Avenue Box 63 Chicago, IL 60611 E-mail: kbarsness@luriechildrens.org 


\section{This article has been cited by:}

1. Yuichi Nishihara, Yoh Isobe, Yuko Kitagawa. 2017. Validation of newly developed physical laparoscopy simulator in transabdominal preperitoneal (TAPP) inguinal hernia repair. Surgical Endoscopy 31:12, 5429-5435. [Crossref]

2. David C. van der Zee. 2017. Endoscopic surgery in children - the challenge goes on. Journal of Pediatric Surgery 52:2, 207-210. [Crossref]

3. Schwab Ben, Rooney Deborah M., Hungness Eric S., Barsness Katherine A.. 2016. Preliminary Evaluation of a Laparoscopic Common Bile Duct Simulator for Pediatric Surgical Education. Journal of Laparoendoscopic \& Advanced Surgical Techniques 26:10, 831-835. [Abstract] [Full Text HTML] [Full Text PDF] [Full Text PDF with Links]

4. Takazawa Shinya, Ishimaru Tetsuya, Harada Kanako, Deie Kyoichi, Fujishiro Jun, Sugita Naohiko, Mitsuishi Mamoru, Iwanaka Tadashi. 2016. Pediatric Thoracoscopic Surgical Simulation Using a Rapid-Prototyped Chest Model and Motion Sensors Can Better Identify Skilled Surgeons Than a Conventional Box Trainer. Journal of Laparoendoscopic \& Advanced Surgical Techniques 26:9, 740-747. [Abstract] [Full Text HTML] [Full Text PDF] [Full Text PDF with Links]

5. Ellen K. O’Brien, Diane B. Wayne, Katherine A. Barsness, William C. McGaghie, Jeffrey H. Barsuk. 2016. Use of 3D Printing for Medical Education Models in Transplantation Medicine: a Critical Review. Current Transplantation Reports 3:1, 109-119. [Crossref]

6. Roy Kazan, Brigitte Courteau, Shantale Cyr, Thomas M. Hemmerling, Mirko Gilardino. 2016. A Novel Mammoplasty Part-Task Trainer for Simulation of Breast Augmentation. Simulation in Healthcare: The Journal of the Society for Simulation in Healthcare 11:1, 60-64. [Crossref]

7. Kanako Harada, Gaku Ishikawa, Shinya Takazawa, Tetsuya Ishimaru, Naohiko Sugita, Tadashi Iwanaka, Mamoru Mitsuishi. 2016. Development of a Neonatal Thoracic Cavity Model and Preliminary Study. Journal of Japan Society of Computer Aided Surgery 18:2, 80-86. [Crossref]

8. Katherine Bootsma, Elizabeth Dimbath, Jason Berberich, Jessica L. Sparks. Materials Used as Tissue Phantoms in Medical Simulation . [Crossref]

9. Kanako Harada, Shinya Takazawa, Yusuke Tsukuda, Tetsuya Ishimaru, Naohiko Sugita, Tadashi Iwanaka, Mamoru Mitsuishi. 2015. Quantitative pediatric surgical skill assessment using a rapid-prototyped chest model. Minimally Invasive Therapy \& Allied Technologies 24:4, 226-232. [Crossref]

10. Barsness Katherine A., Rooney Deborah M., Davis Lauren M., O'Brien Ellie. 2015. Evaluation of Three Sources of Validity Evidence for a Synthetic Thoracoscopic Esophageal Atresia/Tracheoesophageal Fistula Repair Simulator. Journal of Laparoendoscopic \& Advanced Surgical Techniques 25:7, 599-604. [Abstract] [Full Text HTML] [Full Text PDF] [Full Text PDF with Links]

11. Katherine A. Barsness. 2015. Trends in technical and team simulations: Challenging the status Quo of surgical training. Seminars in Pediatric Surgery 24:3, 130-133. [Crossref]

12. Barsness Katherine A., Rooney Deborah M., Davis Lauren M., O'Brien Ellie. 2015. Preliminary Evaluation of a Novel Thoracoscopic Infant Lobectomy Simulator. Journal of Laparoendoscopic \& Advanced Surgical Techniques 25:5, 429-434. [Abstract] [Full Text HTML] [Full Text PDF] [Full Text PDF with Links]

13. Takazawa Shinya, Ishimaru Tetsuya, Harada Kanako, Tsukuda Yusuke, Sugita Naohiko, Mitsuishi Mamoru, Iwanaka Tadashi. 2015. Video-Based Skill Assessment of Endoscopic Suturing in a Pediatric Chest Model and a Box Trainer. Journal of Laparoendoscopic \& Advanced Surgical Techniques 25:5, 445-453. [Abstract] [Full Text HTML] [Full Text PDF] [Full Text PDF with Links]

14. Barsness Katherine A., Rooney Deborah M., Davis Lauren M., O'Brien Ellie. 2015. Evaluation of Three Sources of Validity Evidence for a Laparoscopic Duodenal Atresia Repair Simulator. Journal of Laparoendoscopic \& Advanced Surgical Techniques 25:3, 256-260. [Abstract] [Full Text HTML] [Full Text PDF] [Full Text PDF with Links]

15. Usón-Casaús Jesús, Pérez-Merino Eva María, Rivera-Barreno Ramón, Rodríguez-Alarcón Carlos A., Sánchez-Margallo Francisco M.. 2014. Evaluation of a Bochdalek Diaphragmatic Hernia Rabbit Model for Pediatric Thoracoscopic Training. Journal of Laparoendoscopic \& Advanced Surgical Techniques 24:4, 280-285. [Abstract] [Full Text HTML] [Full Text PDF] [Full Text PDF with Links] 